International Journal of Retina (IJRETINA) 2019, Volume 2, Number 1.

P-ISSN. 2614-8684, E-ISSN.2614-8536

\title{
Management of Rhegmatogenous Retinal Detachment: Tips and Tricks for The Beginners
}

\author{
Lingam Gopal* \\ *National University Health system, Singapore
}

*Correspondence to:

Lingam Gopal,

National University Health

system, Singapore,

gopal_lingam@nuhs.edu.sg

\section{Is it retinal detachment?}

The first step is to determine if the lesion seen in the fundus is elevated retina or other mimicking condition. While most often the diagnosis of retinal detachment is straightforward there could be confusion on occasions. Conditions that can be confused with retinal detachment are retinal edema (as in Berlin's edema); retinoschisis; choroidal detachment; thick vitreous membranes (as in Uveitis), altered blood etc ${ }^{1}$. Evaluation should include careful slit lamp biomicroscopy, indirect ophthalmoscopy and where needed ultrasonography (even when there is view of some fundus details). In eyes with hazy media, it is the combination of information from ophthalmoscopic evaluation, IOP, and ultra sound features that guide the clinician as to the true nature of the condition.

\section{Is it rhegmatogenous retinal detachment?}

Once the presence of retinal elevation is confirmed, the next is to know whether it is caused by a break or not. In most cases the rhegmatogenous nature of retinal detachment is obvious from the appearance of the retinal detachment as well as the presence of visible retinal break.

There are however situations when it may not be that obvious.

1. Chronic retinal detachments can on occasion have some tendency towards shifting fluid, lack the characteristic undulations and can be confused with secondary retinal detachments.

2. Retinal detachments in the milieu of uveitis can sometimes cause confusion since a retinal break can occur due to the traction caused by the vitreous membranes (due to uveitis) and can actually lead to rhegmatogenous retinal detachment. If the break is not obvious, it may be wrongly identified as exacerbated inflammation and treated with enhanced steroids and immunosuppression. Indicators as to the true nature of the problem are- relatively quiet eye; undulations on retinal surface; the extent of retinal detachment following Lincoff's rules; pigment in vitreous cavity etc. Once again, no one sign can be sine qua non. A high index of suspicion and a thorough evaluation are needed to confirm the rhegmatogenous nature of the problem.

3. There are several instances of retinal detachments secondary to tumors such as melanoma having been subjected to scleral buckling. This error in judgment can occur especially, when the surgeon did not evaluate the eye in detail before surgery and relied upon the input from less experienced personnel. This is one instance where ultra sonography is valuable even in the presence of clear media. A tumor underlying the retinal detachment is missed mostly when the examination is very casual.

\section{Discussion with patient/relatives:}

In the discussion with the patient, it is important if one is able to indicate broadly the approach to surgery; the expected success in terms of reattachment of retina; the expected recovery of vision (if surgery is successful); any limitations in travel; risks of surgery including risk of loss of existing vision (especially relevant when operating on eyes with attached macula); need for multiple surgeries (if anticipated) etc. The patients often misinterpret expression of 
percentage of success as the expected visual improvement. This can become very important while operating on eyes with chronic retinal detachments where anatomical success does not translate into visual success.

\section{Planning the approach:}

There is no confusion in case of obviously complicated cases where in vitreo retinal surgery would be the only approach possible such as in cases of gross proliferative vitreo retinopathy(PVR), giant retinal tears, posterior retinal tears, macular holes with retinal detachment, choroidal colobomas with retinal detachment, vitreous hemorrhage with retinal detachment etc.

In case of simple retinal detachments, the options one has are between pneumatic retinopexy, scleral buckling and pars plana surgical approach. A lot would depend on one's training and individual discretion. The following guidelines may be helpful if one has an open mind to choose between the options:

a) If choosing pneumatic retinopexy, please make sure that the entire retina can be evaluated well with binocular indirect ophthalmoscope and scleral indentation to exclude additional retinal breaks - especially inferiorly. Even minimal vitreous hemorrhage or peripheral cortical cataract can interfere with proper visualization all round. Just because there is an obvious horseshoe tear superiorly, does not mean there are no other less obvious breaks elsewhere. Failure to identify the additional breaks is the most common cause for failure of pneumatic retinopexyunfairly blamed on new breaks/ PVR etc.

b) Avoid pneumatic retinopexy in relatively large breaks. Chances of sub retinal migration of the bubbles are high.

c) Retinal detachments due to retinal dialysis are mostly managed with scleral buckling. There are several advantages to managing them with scleral buckling instead of performing vitreo retinal surgery. Most of these are young individuals with no posterior vitreous detachment, and clear lenses.

d) Retinal detachments clearly related to lattice degeneration with atrophic holes are again best managed with scleral buckling. Even eyes with some amount of intra retinal and sub retinal gliosis can do well with scleral buckle in these circumstances.

e) Pseudophakic eyes with retinal detachment: Most pseudophakic detachments are managed by pars plana route. Since iatrogenic cataract is not an issue, pars plana route permits a more through vitrectomy and gas tamponade. The role of encirclage is also grossly reduced when the vitreous is more diligently excised.

f) Addition of encirclage to pars plana surgery would be occasionally needed in eyes where vitreous cannot be closely shaved (as in phakic eyes) along with presence of inferior retinal breaks that could potentially lift up because of contracture of residual vitreous. In the presence of a broad vitreous base and significantly condensed and partly fibrotic peripheral vitreous, adding an encirclage will reduce the risk of recurrent retinal detachment.

g) Addition of encirclage to scleral buckling: Segmental buckles need more closely spaced sutures compared to buckles with encirclage. In the absence of an encirclage, the two extreme ends of the buckle tend to lift up beyond the mattress suture and are effectively not indenting the eye wall.

However, most cases of retinal detachments due to lattice related atrophic holes can be managed by segmental buckling without encirclage. Where the lesions are in several quadrants, it is preferable to place an encircling band in addition to produce a more uniform and sustained indentation.

In the condition of a retinal dialysis, the edges of the dialysis may remain uncovered due to similar reasons and hence it is recommended to add an encircling element.

h) Choice of gas tamponade after vitrectomy: Simple rhegmatogenous retinal detachments with superior breaks distributed in not more than one quadrant can be managed with short acting gases such as SF6. Eyes with wider distribution of breaks or relatively inferior breaks are best managed with longer acting gases such as $\mathrm{C}_{2} \mathrm{~F}_{6}$ or $\mathrm{C}_{3} \mathrm{~F}_{8}$.

\section{Importance of preoperative detailed evaluation}

One cannot over emphasize the need for detailed preoperative evaluation. A detailed retinal drawing is optional but valuable- especially when scleral buckling is performed. The conditions during the surgery may not be as good as in the clinic from the perspective of indirect ophthalmoscopic visualization. In addition to the physical impediments caused by the operation theatre set up, trolleys and sterile draping, the cornea can become edematous and the pupil may constrict causing sub optimal visualization. The presence of a detailed drawing can function as an excellent road map to help localize the lesions under these circumstances. In addition, the act of performing a detailed retinal drawing forces the novice surgeon to become familiar with the retinal condition so well that surgical time can actually be shortened. 


\section{Issues that could potentially compromise the final outcome}

1. Decision to do scleral buckling when the visualization is inadequate.

2. Attracted by one obvious large tear and missing the other tiny tears along the vitreous base.

3. DACE technique (Drain- Air injection- Cryo- Exoplant) that has gone awry: Too many bubbles of air that interfere with proper localization of break as well as appropriateness of the buckle location.

4. Not evaluating the location of the break Vs buckle relationship at conclusion (after drainage). In some cases the break may fall just at the edge of buckle and may not be adequately supported. If identified during surgery, what is needed is a small additional step of shifting the buckle posteriorly to avoid recurrences.

5. Not placing an adequately wide buckle: There is a general tendency to choose the buckles with narrow width (\#276/277) since it makes it easier to place the mattress sutures. However this choice should not be at the expense of inadequate coverage of the break. One should not hesitate to place a broader buckle such as \#279 if needed.

6. Sub retinal bleed at conclusion of SRF drainage: This is not an often-predictable problem. Contrary to intuitive thinking, significant bleeds can occur at conclusion of drainage even in younger age groups and in non-myopic eyes. Cauterization of the knuckle of choroid does not always prevent the bleed. Most significant bleeds occur at conclusion of drainage when the pressure is released suddenly- even before the sutures can be tightened. One way out can be to do controlled drainage. Drain some amount- inject BSS- drain more and then tighten the buckle sutures. If despite the precautions bleed occurs and has migrated into sub retinal space, one can inject a bubble of $\mathrm{C}_{3} \mathrm{~F}_{8}$ and place patient prone to permit the blood to shift away from macula.

7. Inadequate cryo induced chorio retinal adhesion around the retinal break: This situation can arise when the retina in the area of break is highly elevated and the ice ball formed by the cryo probe is not reaching the retina. Theoretically freezing the choroid- RPE complex should be enough to cause adequate chorio retinal adhesion once the retina falls on the treated RPE. However due to the high degree of parallax the freezing of the choroid/RPE may not be at the right place- as desired.

In such cases, one can supplement with post-operative laser as long as there is no recollection of fluid around the break.

8. Fish mouthing: This is not an uncommon complication after placing circumferential buckles for large horseshoe tears. Injecting a bubble of gas and positioning the patient can easily remedy this complication.

\section{The use and misuse of silicone oil:}

In addition to more common indications such as severe PVR, coloboma related retinal detachments, large giant retinal tears ( 180 degrees and more) etc, one may have to use silicone oil in patients who cannot posture themselves, patients who need to fly immediately after surgery, and one eyed patients (for early visual rehabilitation).

One should not take usage of silicone oil lightly. Once injected, we have committed the patient for at least one more surgery. The worry of facing a recurrent total retinal detachment after gas tamponade should not push novice surgeons to over use of silicone oil. With use of silicone oil, we post pone the issues but do not get rid of them. Long-term complications are well known. ${ }^{2}$ Shallow inferior recurrent retinal detachments may go unnoticed.

There is a tendency to postpone removal of silicone oil even when retina is well attached - for fear of facing possible recurrence after removal of silicone oil. Some surgeons keep convincing themselves that it is OK to leave the oil in till it causes complications. Some surgeons place silicone oil for inferior breaks, where gas tamponade with additional encirclage would have sufficed- just to avoid converting so called 'suture less vitrectomy' to a sutured one.

\section{Adopting new techniques:}

Change is a constant phenomenon and this applies to the field of vitreo retinal surgery as well. Adopting a new technique does not mean one should forget an old technique- especially one as robust as scleral buckling. It is accepted that scleral buckling is less forgiving but the tendency to manage all cases of retinal detachment with pars plana approach should be abhorred.

The new technique of using a $25 \mathrm{G}$ chandelier light along with the wide-angle visualization system (BIOM etc) and performing scleral buckling has certain advantages. ${ }^{3}$ Unlike with indirect ophthalmoscopy, the image of the retina can be magnified making it easier to localize smaller lesions. Use of this hybrid technique may be the best way of bringing some of the young surgeons back to fold of scleral buckling. 


\section{REFERENCES:}

1. Bhomaj S Khare C. Retinal detachment - in Post graduate ophthalmology. Editors- Zia Chaudhari, Murugesan Vanathi. Jaypee highlights, New Delhi. Chapter-12.11; Page1255

2. Federman JL, Schubert HD. Complications associated with the use of silicone oil in 150 eyes after retina-vitreous surgery. Ophthalmology. 1988;95(7):870-876.

3. Temkar S. Takkar B, Azad SV, Venkatesh P. Endoillumination (chandelier) assisted scleral buckling for a complex case of retinal detachment. Ind J ophthalmol 2016;64:845-6. 